\title{
Recycling NSs to ultrashort periods: a statistical analysis of their evolution in the $\mu-P$ plane
}

\author{
Andrea Possenti \\ Dip. Astronomia dell'Università, via Ranzani 1, 40127 Bologna, Italy \\ Monica Colpi \\ Dip. Fisica Mi-Bicocca, P.za della Scienza 3, 20126 Milano, Italy \\ Ulrich Geppert \\ AIP, An der Sternwarte 16, 14482 Potsdam, Germany \\ Luciano Burderi \\ Oss. Astronomico di Monteporzio, via Frascati 33, 00044 Roma, Italy
}

Nichi D'Amico

Oss. Astronomico di Bologna, via Ranzani 1, 40127 Bologna, Italy

\begin{abstract}
We investigate the statistical evolution of magnetic neutron stars recycled in Low Mass Binary (LMB) systems, simulating synthetic populations. Irrespective to the details of the physical models, we find to be significant the fraction of neutron stars spinning close to their mass shedding limit relative to the millisecond pulsar population. The simulated neutron stars show a tail in their period distribution at periods shorter than $1.558 \mathrm{~ms}$, the minimum detected so far. Crustal magnetic field decay models predict also the existence of massive rapidly spinning neutron stars with very low magnetic moment.
\end{abstract}

\section{Magnetorotational evolution scenario}

Population synthesis models are evolved within a simple recycling scenario (as outlined e.g. by Lipunov 1992), where the neutron stars (NSs hereon) may experience the phase of ejector, accretor or propeller. The magnetorotational evolution includes the general relativistic effects as modelled in Burderi et al. (1999). We considered two possibilities bracketing uncertainties in the accretion histories of the NSs in LMBs: (i) steady accretion for a time $\tau_{R L O}$; (ii) persistent accretion followed by a transient phase during which the mass transfer becomes unsteady, mimicking the quenching of accretion (Ergma et al. 1998). In particular we account for the angular momentum losses by propeller, both for persistent and for non-stationary accretion, during the Roche Lobe Overflow phase.

The NSs in our population synthesis models have an initial gravitational mass of $1.4 \mathrm{M}_{\odot}$. Cook, Shapiro \& Teukolsky (1994) showed that all viable equations of state (EoSs) for nuclear matter allow for recycling to ultra-short periods, 
in the case of a nonmagnetic NS. The minimum period below which the massshedding instability limit is encountered, depends however on the equation of state. We consider either a soft (FP) and a stiff (PS) EoS, whose critical periods for mass-shedding are $P_{s h}=0.73 \mathrm{~ms}$ (for FP-EoS) and of $P_{s h}=1.40 \mathrm{msec}$ (for PS-EoS), respectively.

A basic assumption for the present investigation is the crustal origin of the NS magnetic field. Its subsequent evolution is governed by the induction equation for ohmic diffusion and advection (see Urpin et al. 1998). While at the surface the standard boundary condition for a dipolar field applies, the inner boundary condition is subject of scientific debates. Hence we explored two hypotheses, imposing at the crust-core boundary either complete field expulsion by the superconducting core (Boundary condition I, BC I) or advection and freezing (Konar \& Bhattacharya 1997) in a very highly conducting transition shell (BC II).

In paper I, Possenti et al. (1998) evolved NSs in low mass binaries (LMBs) through the five phases of the recycling scenario, and explored the evolution of either a core field threaded in the proton fluxoids, and a surface field buried by the diamagnetic accreting plasma. Here, we start with a population of NSs at the onset of the Roche Lobe Overflow (RLO) phase and include the physical evolution of a crustal field. The adopted parameters are:

\begin{tabular}{|c|c|c|c|}
\hline Physical quantity & Distribution & Values & Units \\
\hline NS period at $t_{0}^{\mathrm{RLO}(a)}$ & Flat & 100 & $\sec$ \\
\hline NS $\mu$ at $\mathrm{t}_{0}^{\mathrm{RLO}}(a)$ & Gaussian & $\log <\mu_{0}>=28.50 ; \sigma=0.32$ & $\mathrm{G} \mathrm{cm}^{3}$ \\
\hline$\dot{m}$ in RLO phase ${ }^{(b)}$ & Gaussian & $\log \langle\dot{m}\rangle=-1.00 ; \quad \sigma=0.50$ & $\dot{\mathrm{M}}_{\mathrm{E}}$ \\
\hline Minimum accreted mass & One-value & 0.01 & $\mathbf{M}_{\odot}$ \\
\hline RLO accretion time $(c)$ & Flat in $\log$ & $\rightarrow \tau_{R L O}^{\max }(d)$ & year \\
\hline MSP phase time & Flat in Log & $\rightarrow \quad 3 \times 10^{9}$ & year \\
\hline
\end{tabular}

(a) $\mathrm{t}_{0}^{\mathrm{RLO}}=$ initial time of the RLO phase

(b) baryonic accretion rate during the RLO phase

(c) a Maximum accreted Mass of $0.5 M_{\odot}$ is permitted during the RLO phase

(d) max duration of the RLO phase; typical values: $5 \times 10^{7} \mathrm{yr}-10^{8} \mathrm{yr}-5 \times 10^{8} \mathrm{yr}$

The values in this Table are derived partly from the observation of LMXBs and partly from the tracks in the $\mu-P$ plane calculated by Urpin, Geppert \& Konenkov (1998) for a sample of pulsars, under different hypotheses for the binary evolution times, for the $\mu$ decay and for the angular momentum transfer at the magnetospheric radius.

\section{Results and Discussion}

To execute the synthesis calculation we built a Monte Carlo code, using typically 3,000 particles. The statistical analysis is carried on only those NSs reaching the so-called "millisecond strip" at the end of recycling, the ones having period $P \leq 10.0 \mathrm{~ms}$ and whichever value of the magnetic moment $\mu$. In accordance with the values of $P_{\min }$ and $\mu_{\min }$ (the weakest magnetic moment observed; $\mu_{\text {min }}=7.3 \times 10^{25} \mathrm{G} \mathrm{cm}^{3}$ ), we divided our particles in four groups. Those filling the first quadrant in the millisecond strip $\left(P \geq P_{\min }\right.$ and $\left.\mu \geq \mu_{\min }\right)$ behave as the known MSPs. Also the objects belonging to the second quadrant $\left(P<P_{\min }\right.$ and $\mu \geq \mu_{\text {min }}$ ) should shines as pulsars (see Burderi \& D'Amico 1997). The observability of the objects in the third quadrant $\left(P<P_{\min }\right.$ and $\left.\mu<\mu_{\min }\right)$ as 

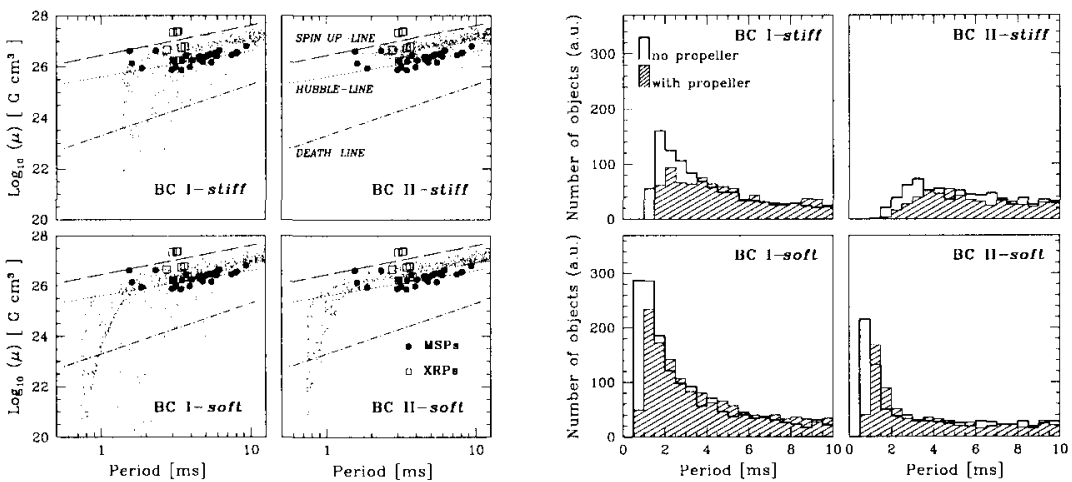

Figure 1. Left: Statistical properties of the synthesized populations in the $\mu-P$ plane, for $\langle\dot{m}\rangle=0.1$ and $\tau_{R L O}^{\max }=5 \times 10^{8} \mathrm{yr}$. Full dots represent the sample of detected MSPs, while open squares represent the NSs in LMXBs (White \& Zhang 1997). Right: Calculated distributions (with and without propeller) of millisecond NSs as a function of the spin period $P$, while $\mu$ covers the whole range of values.

radio sources represents instead a challenge to the modern pulsar surveys. Most of these NSs will be above the Chen and Ruderman (1993) "dea.th-line", and might have a bolometric luminosity comparable to that of the known MSPs. Thereafter we shortly refer as sub-MSPs to all the objects having $P<P_{\text {min }}$ and $\mu$ above the "death-line". Objects in the fourth quadrant $\left(P \geq P_{\min }\right.$ and $\mu<\mu_{m i n}$ ) are probably radio quiet neutron stars (RQNSs), because they tend to be closer to the theoretical "death-line", and they are in a period range which was already searched with good sensitivity by the radio surveys.

The left panel of Figure 1 illustrates how the simulated NSs fill the $\mu-P$ plane, while the right panel shows how the EoS affects the shape of the period distribution. Figure 2 reports on the typical relative abundances (left) and gives indication on the role played by the propeller (right) in modifying the results.

\section{Conclusions}

The population synthesis calculation leads to a number of interesting predictions:

A. It shows the presence of a tail in the period distribution of the simulated NS populations at periods shorter than $1.558 \mathrm{~ms}$.

B. The fraction of $s u b$-MSPs estimated over the entire MSPs population varies between 0 to $\simeq 50 \%$. The EoS and the parameters for the recycling play both an important role in determining these percentages.

C. For a mild-soft EoS and irrespective to the boundary condition at the crust-core interface for the magnetic field evolution, the recycling in LMBs gives rise to a NS distribution increasing toward short periods and a clear barrier is present at the minimum period for mass-shedding.

D. For a stiff EoS, the distribution is flatter and displays a broad maximum. The maximum is located where most of the spin periods of the NSs in LMXBs 


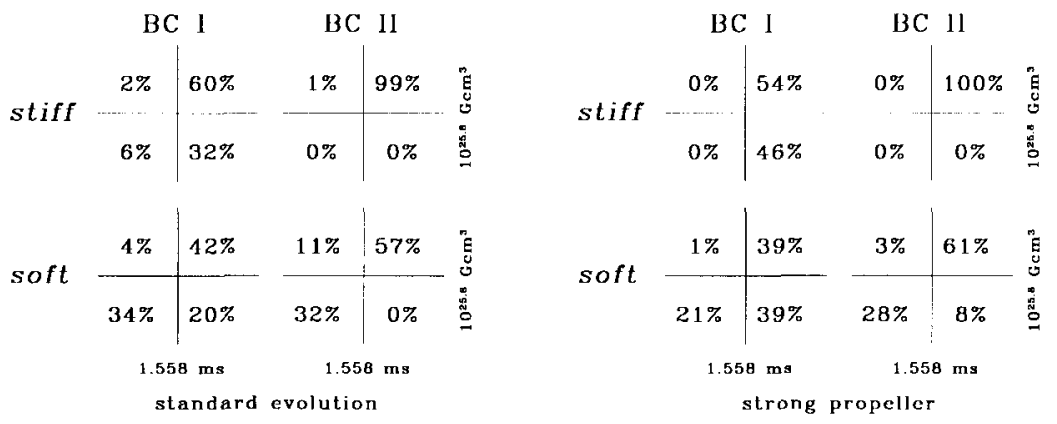

Figure 2. Distributions of the synthesized NSs, derived normalizing the sample to the total number of stars with $P<10 \mathrm{~ms}$. We have divided the $\mu-P$ plane in four regions. The upper left number in each cross gives the percentage of objects having $P\left\langle P_{\min }\right.$ and $\left.\mu\right\rangle$ $\mu_{\text {min }}$. Left: Distribution for the parameters of Fig. 1-left. Right: Distributions when a strong propeller is applied, as in Fig. 1-right.

are found, as indirectly inferred form the quasi periodic oscillations seen in the timing analysis of their X-ray light curves.

E. If NSs at the end of persistent accretion in a LMB experience a phase of smooth decline of the accretion rate, the magnetospheric propeller at the end of the RLO phase produces a depletion of fastly spinning NSs but, at least for the soft EoS, it preserves a distribution that peaks at periods $\sim 1.5 \mathrm{~ms}$.

$\mathbf{F}$. The models for the decay of a crustal magnetic field predict the existence of spun up NSs with very low magnetic moment: their period distribution is a neat signature for the physics at the crust-core interface.

This investigation shows that the detection of $s u b$-MSPs represents a serious challenge to modern radio searches.

\section{References}

Burderi, L., \& D'Amico, N. 1997, ApJ, 490, 343

Burderi, L., Possenti, A., Colpi, M., Di Salvo, T., D’Amico, N. 1999, ApJ, 519, 285

Chen, K., \& Ruderman, M. 1993, ApJ, 402, 264

Cook, G.B., Shapiro S.L., \& Teukolsky, S.A. 1994, ApJ, 423, L117

Ergma, E., Sarna, M.J. \& Antipova, J. 1998, MNRAS, 300, 352

Konar, S., \& Bhattacharya, D., 1997, MNRAS, 284, 311

Lipunov, V.M. 1992, in Astrophysics of neutron stars, Springer, Berlin

Possenti, A., Colpi, M., D'Amico, N., \& Burderi, L. 1998, ApJ, 497, L97

Urpin, V.A., Geppert U., \& Konenkov, D. 1998, MNRAS, 295, 907

White, N.E., \& Zhang, W. 1997, ApJ, 490, L87 